\title{
Strategy Proof Mechanisms for Facility Location at Limited Locations
}

\author{
Toby Walsh \\ UNSW Sydney and CSIRO Data61
}

\begin{abstract}
Most studies of facility location problems permit a facility to be located at any position. In practices, this may not be possible. For instance, we might have to limit facilities to particular locations such as at highway exits, close to bus stops, or on empty building lots. We consider here the impact of such constraints on the location of facilities on the performance of strategy proof mechanisms for facility location. We study six different objectives: the total or maximum distance agents must travel, the utilitarian or egalitarian welfare, and the total or minimum satisfaction of agents (satisfaction is a normalized form of utility). We show that limiting the location of a facility makes all six objectives harder to approximate. For example, we prove that the median mechanism modified suitably to locate the facility only at a feasible location is strategy proof and 3-approximates both the optimal total distance and the optimal maximum distance. In fact, this is optimal as no deterministic and strategy proof mechanism can better approximate the total or maximum distances. This contrasts with the setting where the facility can be located anywhere, and the median mechanism returns the optimal total distance and 2-approximates the maximum distance.
\end{abstract}

\section{Introduction}

The facility location problem has been studied using tools from a wide variety of fields such as AI (e.g. [1,2]). Operations Research (e.g. [3,4]), and Game Theory (e.g. [5,6]). Our goal here is to design mechanisms that locate the facility in a way that the agents have no incentive to mis-report their true locations. Facility location models many practical problems including locating bus or tram stops, schools, playgrounds, telephone exchanges, mobile phone masts, recycling centres, electric car charging locations, shared cars, power plants, electricity substations, doctors, chemists, fire stations, and hospitals to serve a community. In many of these real world settings, facilities may be limited in where they can be located. For example, a warehouse might need to be constrained to be near to the railway, or an ambulance station close to a highway. Our contribution is to demonstrate that such constraints on the location of a facility make it harder to design strategy proof mechanisms which provide high quality solutions. We measure the quality of the solution in six different ways: total or maximum distance of the agents to the facility, the utilitarian and egalitarian welfare, and the social or minimum satisfaction.

From a technical sense, limiting the location of a facility might appear to change little the facility location problem. We merely need to limit the space of mechanisms to the strict subset of mechanism which only locate facilities at feasible locations. We can therefore immediately inherit many impossibility results. For instance, since there is no 
deterministic and strategy proof mechanism for the facility problem which minimizes the maximum distance an agent travels when the facility can be located anywhere, it follows quickly that there is no such mechanism when the facility is limited in its location. The mechanisms excluded because they locate facilities at infeasible locations are often precisely those with good normative properties. Our contribution here is to show restricting mechanisms to locate facilities only at feasible locations often increases approximation ratios, irrespective of whether the objective is distance, welfare or satisfaction. However, the extent to which approximation ratios increases depends very much on the objective and the problem. For example, the lower bound on the best possible approximation ratio of the optimal egalitarian welfare increases from $\frac{3}{2}$ to unbounded when we limit the feasible location of a facility. On the other hand, the best possible approximation ratio of the optimal utilitarian welfare only triples in this case. Our results are summarized in Tables 1 and 2.

\begin{tabular}{|c||c|c|c|c|c|c|}
\hline measure & $\begin{array}{c}\text { total } \\
\text { distance }\end{array}$ & $\begin{array}{c}\text { max } \\
\text { distance }\end{array}$ & $\begin{array}{c}\text { utilitarian } \\
\text { welfare }\end{array}$ & $\begin{array}{c}\text { egalitarian } \\
\text { welfare }\end{array}$ & $\begin{array}{c}\text { social } \\
\text { satisfaction }\end{array}$ & $\begin{array}{c}\text { min } \\
\text { satisfaction }\end{array}$ \\
\hline \hline lower bound & $3[1]$ & $\mathbf{3}[2]$ & $\mathbf{3}[1]$ & $\infty\left[\frac{3}{2}\right]$ & $\infty[1.07]$ & $\infty\left(\frac{4}{3}\right)$ \\
\hline MEDIAN $^{*}$ & $3[1]$ & $\mathbf{3}[2]$ & $\mathbf{3}[1]$ & $\infty[\infty]$ & $\infty\left[\frac{3}{2}\right]$ & $\infty[\infty]$ \\
\hline \hline
\end{tabular}

Table 1: Approximation ratios achievable by deterministic and strategy proof mechanisms for the single facility location problem at limited locations. Bold for results proved here. [Numbers] in brackets are the approximation ratios achieved for when the facility can be located anywhere.

\begin{tabular}{|c||c|c|c|c|c|c|}
\hline measure & $\begin{array}{c}\text { total } \\
\text { distance }\end{array}$ & $\begin{array}{c}\text { max } \\
\text { distance }\end{array}$ & $\begin{array}{c}\text { utilitarian } \\
\text { welfare }\end{array}$ & $\begin{array}{c}\text { egalitarian } \\
\text { welfare }\end{array}$ & $\begin{array}{c}\text { social } \\
\text { satisfaction }\end{array}$ & $\begin{array}{c}\text { min } \\
\text { satisfaction }\end{array}$ \\
\hline ENDPOINT $^{*}$ & $2 \mathrm{n}-3[\mathrm{n}-2]$ & $3[2]$ & $\mathbf{2}[2]$ & $\frac{3}{2}\left[\frac{3}{2}\right]$ & $\frac{3 n}{4}-\frac{1}{2}\left[\frac{n}{2}-\frac{1}{4}\right]$ & $\infty[\infty]$ \\
\hline
\end{tabular}

Table 2: Summary of approximation ratios achieved by ENDPOINT* mechanism for the two facility location problem at limited locations. Bold for results proved here. (Numbers) in brackets are the approximation ratios achieved by the corresponding ENDPOINT mechanism when the two facilities can be located anywhere.

\section{Related work}

We follow the line of work initiated by Procaccia and Tennenholtz [1] that looks to resolve the inherent tension in designing mechanisms that are strategy proof and effective by identifying strategy proof mechanisms that are guaranteed to return solutions within some constant factor of optimal. The most related prior work to ours is by Feldman, Fiat, Golomb [7]. This also considers facility location problems where the 
facility is restricted to limited locations. There is, however, a critical difference with this work. This earlier work restricted analysis to a single objective (sum of distances), while here we consider six objectives (sum/maximum distance, utilitarian/egalitarian welfare, social/minimum satisfaction). Our results show that approximation ratios that can be achieved depend critically on the objective chosen. For instance, when the facility is restricted to limited locations, deterministic and strategy proof mechanisms can 3 -approximate the optimal utilitarian welfare. However, no deterministic and strategy proof mechanism has a bounded approximation ratio for the egalitarian welfare. By contrast, if we consider a different but related objective to the egalitarian welfare such as the maximum distance an agent travels, then deterministic and strategy proof mechanisms exist which bound the approximation ratio even when the facility is restricted to limited locations. In addition, when the facility is unrestricted, there exists a deterministic and strategy proof mechanism that can $\frac{3}{2}$-approximate the optimal egalitarian welfare. The choice of objective then reveals different aspects of the approximability of these facility location problems.

One month after this work here first appeared as a preprint, Tang, Wang, Zhang and Zhao published a preprint looking independently at a special case of this problem in which facilities are limited to a finite set of locations [8]. There are two significant technical differences between the two studies. First, the work of Tang et al. does not capture the more general setting here where the facility is limited to a set of subintervals. In their work, a facility is limited to a finite set of locations. Their model cannot then describe a setting where, for example, a school must be within $500 \mathrm{~m}$ of one of the neighbourhood bus stops as the feasible set is not finite. In addition, the work of Tang et $a l$. , like the work of Feldman et al., only considers two objectives: total and maximum cost. Here we consider four additional objectives: utilitarian and egalitarian welfare, as well as social and minimum satisfaction. Our results show that we can achieve very different approximation ratios with these different objectives. Indeed, for many of these new objectives, we cannot achieve a bounded approximation ratio with deterministic and strategy proof mechanisms.

As in much previous work on mechanism design for facility location (e.g. [1]), we consider the one-dimensional setting. This models a number of real world problems such as locating shopping centres along a highway, or ferry stops along a river. There are also various non-geographical settings that can be viewed as one-dimensional facility location problems (e.g. choosing the temperature of a classroom, or the tax rate for property transactions). In addition, we can use mechanisms for the one-dimensional facility location problem in more complex settings (e.g. we can decompose the 2-d rectilinear problem into a pair of 1-d problems). Finally, results about mechanisms for the one-dimensional problem can inform the results about mechanisms for more complex metrics. For instance, lower bounds on the performance of strategy proof mechanisms for the 1-d problem provide lower bounds for the 2-d problem.

\section{Formal background}

We have $n$ agents located on $[0,1]$, and wish to locate one or more facilities also on $[0,1]$ to serve all the agents. Agent $i$ is at location $x_{i}$. Without loss of generality, we suppose 
agents are ordered so that $x_{1} \leq \ldots \leq x_{n}$. A solution is a location $y_{j}$ for each facility $j$. Agents are served by their nearest facility. We consider six different performance measures: total or maximum distance, utilitarian or egalitarian welfare, and social or minimum satisfaction.

The total distance is $\sum_{i=1}^{n} \min _{j}\left|x_{i}-y_{j}\right|$. The maximum distance is $\max _{i=1}^{n} \min _{j} \mid x_{i}-$ $y_{j} \mid$. We suppose the utility $u_{i}$ of agent $i$ is inversely related to its distance from the facility serving it. More precisely, $u_{i}=1-\min _{j}\left|x_{i}-y_{j}\right|$. Utilities are, by definition, in $[0,1]$. The utilitarian welfare is the sum of the utilities of the individual agents, $\sum_{i=1}^{n} u_{i}$. The egalitarian welfare is the minimum utility of any agent, $\min _{i=1}^{n} u_{i}$.

In $[9,10]$, normalized utilities called "happiness factors" are introduced. The happiness $h_{i}$ of agent $i$ is $h_{i}=1-\min _{j} \frac{\mid x_{i}-y_{j}}{d_{\max }^{i}}$ where $d_{\text {max }}^{i}$ is the maximum possible distance agent $i$ may need to travel. Here $d_{\max }^{i}=\max \left(x_{i}, 1-x_{i}\right)$. Note that the happiness of an agent is, by definition, in $[0,1]$. The social satisfaction is then the sum of the happinesses of the individual agents, $\sum_{i=1}^{n} h_{i}$. The minimum satisfaction is the minimum happiness of any agent, $\min _{i=1}^{n} h_{i}$. Our goal is to optimize one of the distance, welfare or satisfaction objectives.

We consider some particular mechanisms for facility location. Many are based on the function median $\left(z_{1}, \ldots, z_{p}\right)$ which returns $z_{i}$ where $\left|\left\{j \mid z_{j}<z_{i}\right\}\right|<\left\lceil\frac{p}{2}\right\rceil$ and $\left|\left\{j \mid z_{j}>z_{i}\right\}\right| \leq\left\lfloor\frac{p}{2}\right\rfloor$. With $n-1$ parameters $z_{1}$ to $z_{n-1}$ representing "phantom" agents, a GENMEDIAN mechanism locates the facility at median $\left(x_{1}, \ldots, x_{n}, z_{1}, \ldots, z_{n-1}\right)$ As we argue shortly, such mechanisms characterize an important class of strategy proof mechanisms [11]. The LEFTMOST mechanism has parameters $z_{i}=0$ for $i \in[1, n)$ and locates the facility at the location of the leftmost agent. The RiGHTMOST mechanism has parameters $z_{i}=1$ for $i \in[1, n)$ and locates the facility at the location of the rightmost agent. The MEDIAN mechanism has parameters $z_{i}=0$ for $i \leq\left\lfloor\frac{n}{2}\right\rfloor$ and 1 otherwise, and locates the facility at the median agent if $n$ is odd, and the leftmost of the two median agents if $n$ is even. The MidORNEAREST mechanism is an instance of GENMEDIAN with parameters $z_{i}=\frac{1}{2}$ for $i \in[1, n)$, locating the facility at $\frac{1}{2}$ if $x_{1} \leq \frac{1}{2} \leq x_{n}$, and otherwise at the nearest $x_{i}$ to $\frac{1}{2}$. The ENDPOINT mechanism locates one facility with the LEFTMOST mechanism and another with the RIGHTMOST mechanism.

We extend this model of facility location problems with constraints on the location of the facility. In particular, we suppose the interval $[0,1]$ is decomposed into a set of feasible and disjoint (open or closed) sub-intervals, and the facility must be located within one of these sub-intervals. Our goal is to see how restricting the feasible locations of the facility in this way impacts on the performance of strategy proof mechanisms. Note that unlike [7], agents are not limited in where they can be located. We only limit where the facility (and not agents) can be located. In particular, we modify mechanisms to ensure the facility is located at a feasible location. For instance, the LEFTMOST* mechanism modifies the LEFTMOST mechanism to locate the facility at the nearest feasible location to the leftmost agent. The RIGHTMOST* mechanism modifies the RIGHTMOST mechanism in a similar fashion. Note that we cannot have agents simply report their nearest feasible location as there might be a choice of such locations. Indeed, many of our results that approximation guarantees are not bounded arise 
because of the difficulty of choosing between the two nearest and feasible locations to some optimal but infeasible facility location in a strategy proof way.

We consider three desirable properties of mechanisms: anonymity, Pareto optimality and strategy proofness. Anonymity is a fundamental fairness property that requires all agents to be treated alike. Pareto optimality is one of the most fundamental normative properties in economics. It demands that we cannot improve the solution so one agent is better off without other agents being worse off. Finally, strategy proofness is a fundamental game theoretic property that ensures agents have no incentive to act strategically and try to manipulate the mechanisms by mis-reporting their locations.

More formally, a mechanism is anonymous iff permuting the agents does not change the solution. A mechanism is Pareto optimal iff it returns solutions that are always Pareto optimal. A solution is Pareto optimal iff there is no other solution in which one agent travels a strictly shorter distance, and all other agents travel no greater distance. A mechanism is strategy proof iff no agent can mis-report and thereby travel a shorter distance. For instance, the MEDIAN mechanism is anonymous, Pareto optimal and strategy proof. Finally, we will consider strategy proof mechanisms that may approximate the optimal distance, welfare or satisfaction. A mechanism achieves an approximation ratio $\rho$ iff the solution it returns is within a factor of $\rho$ times the optimal. In this case, we say that the mechanism $\rho$-approximates the optimal.

Procaccia and Tennenholtz initiated the study of designing approximate and strategy proof mechanisms for locating facilities on a line [1]. With just one facility, they argue that the MEDIAN mechanism is strategy proof and optimal for the total distance, while the LEFTMOST mechanism is strategy proof and 2-approximates the optimal maximum distance, and no deterministic and strategy proof mechanism can do better.

\section{Single facility, distance approximations}

For a single facility on the line, Moulin proved a seminal result that any mechanism that is anonymous, Pareto optimal and strategy proof is a generalized median mechanism, GenMedian [11]. This locates the facility at the median location of the $n$ agents and $n-1$ "phantom" agents. We cannot apply Moulin's result directly to our setting as a GENMEDIAN mechanism may select an infeasible location for the facility. Instead, we consider the GENMEDIAN* mechanism which locates a facility at the nearest feasible location to that returned by a GENMEDIAN mechanism. If there are two nearest and equi-distant feasible locations, then the GENMEDIAN ${ }^{*}$ mechanism uses a fixed tiebreaking rule for each infeasible interval (e.g,. always use the leftmost of the two nearest locations). Here, as indeed throughout the paper, we suppose a fixed tie-breaking rule to ensure that the modified mechanism retains anonymity and strategy proofness. However, none of our results on performance guarantees depend on the choice.

The MEdian* mechanism is an instance of GENMEDIAN* which locates the facility at the median agent if it is a feasible location, and otherwise at the nearest feasible location to the median agent. Massó and Moreno de Barreda prove that, when locating a single facility at limited locations, a mechanism is anonymous, Pareto efficient and strategy proof iff it is a GENMEDIAN* mechanism with at least one phantom agent at 
0 and one at 1 (corollary 2 in [12]). It follows that the MEDIAN* mechanism is anonymous, Pareto efficient and strategy proof.

\subsection{Total distance}

We first consider the objective of minimizing the total distance agents travel to be served. The MEDIAN* mechanism 3-approximates the optimal total distance (Lemma 21 in [7]). In fact, this is optimal. No deterministic strategy proof mechanism can better approximate the total distance in general (Lemma 19 in [7]). By comparison, when the facility can be located anywhere, the MEDIAN mechanism is strategy proof and returns the optimal total distance. Limiting the feasible locations of a facility therefore worsens the performance of the best possible deterministic and strategy proof mechanism. In particular, the best possible deterministic and strategy proof mechanism goes from returning an optimal solution to 3-approximating the optimal total distance.

\subsection{Maximum distance}

We consider next the objective of minimizing the maximum distance any agent travels to be served. The MEDIAN* mechanism also 3-approximates the optimal maximum distance.

Theorem 1 The MEDIAN* mechanism 3-approximates the optimal maximum distance for a facility location problem with limited locations.

Proof: There are three cases. In the first case, the median agent is at a feasible location. The most an agent needs to travel to the facility at the median agent is then at most the distance of the rightmost agent from the leftmost. This is at most twice the optimal. Hence, this 2-approximates the maximum distance. In the second case, the median agent is not at a feasible location, and the facility is located to the left at the nearest feasible location to the median agent. There are two sub-cases. In the first sub-case, the optimal location for the facility is at this point or even further to the left. But this means the facility is located between the leftmost and rightmost agents. Hence, this is again at worst a 2-approximation of the maximum distance. in the second sub-case, the optimal location for the facility is to the right, within some feasible interval to the right of the median agent. The worst such sub-case for the approximation ratio is when the only feasible locations for the facility are at 0 or $\frac{2}{3}$, two agents are at $\frac{1}{3}-\epsilon$ with $\frac{1}{3}>\epsilon>0$, and one agent is at 1 . In this sub-case, the MEDIAN* mechanism locates the facility at 0 . This gives a maximum distance of 1 . However, the optimal maximum distance of just $\frac{1}{3}+\epsilon$ requires the facility to be located at $\frac{2}{3}$. The approximation ratio is thus $\frac{3}{1+3 \epsilon}$ which tends to 3 from below as $\epsilon$ approaches zero. The solution returned is therefore at worst a 3-approximation of the optimal maximum distance. The third case locates the facility to the right of the median agent and is symmetric to second case. $\diamond$

We contrast this with the setting where the facility can be located anywhere, and the MEDIAN mechanism 2-approximates the optimal maximum distance. In fact, when there are no constraints on where facilities can be located, the MEDIAN mechanism 
is optimal as no deterministic and strategy proof mechanism can do better than 2approximate the optimal maximum distance (Theorem 3.2 of [1]). Restricting the feasible locations of a facility therefore worsens the performance of a median mechanism from a 2-approximation of the optimal maximum distance to a 3-approximation.

Can any strategy proof mechanism do better than 3-approximate the maximum distance when we limit the feasible locations of the facility? We show that no deterministic and strategy proof mechanism has a smaller approximation ratio for the optimal maximum distance.

Theorem 2 For a facility location problem with limited locations, no deterministic and strategy proof mechanism can do better than 3-approximate the optimal maximum distance.

Proof: Suppose the only feasible locations for the facility are at $\frac{1}{4}$ or $\frac{3}{4}$, and there are two agents, one at $\frac{1}{2}-\epsilon$ and the other at $\frac{1}{2}+\epsilon$ for $\frac{1}{2}>\epsilon>0$. There are two cases. In the first case, the mechanism locates the facility at $\frac{1}{4}$. Suppose the agent at $\frac{1}{2}+\epsilon$ misreports their location as 1 . Since the mechanism is strategy proof, the location of the facility cannot change. Consider this new problem with one agent at $\frac{1}{2}-\epsilon$ and the other at 1 . The optimal maximum distance is now $\frac{1}{4}+\epsilon$ with the facility at $\frac{3}{4}$. However, the solution returned by the mechanism has a maximum distance of $\frac{3}{4}$. The approximation ratio is $\frac{3}{1+4 \epsilon}$. As $\epsilon$ approaches zero, this tends to 3 from below. Hence the mechanism at best 3 -approximates the total distance. The second case where the facility is located at $\frac{3}{4}$ rather than $\frac{1}{4}$ is symmetric. $\diamond$

Hence the MEDIAN* mechanism is optimal. No deterministic and strategy proof mechanism can do better than 3-approximate the optimal maximum distance.

\section{Single facility, welfare approximations}

We switch now to considering how well strategy proof mechanisms approximate the utilitarian or egalitarian welfare.

\subsection{Utilitarian welfare}

With no limits on the location of the facility, it is not hard to see that the MEDIAN mechanism is strategy proof and returns the optimal utilitarian welfare. However, in our setting, the MEDIAN mechanism may select an infeasible location for the facility. We consider instead the MEDIAN* mechanism which locates the facility at the nearest feasible location to the median agent.

Theorem 3 For a facility location problem with limited locations, the MEDIAN* mechanism is strategy proof and 3-approximates the optimal utilitarian welfare.

Proof: A worst case is when the only feasible locations for the facility are at 0 or $1, k$ agents are at $\frac{1}{2}$, and $k$ more at 1 . Suppose the tie-breaking rule of the MEdian* mechanism locates the facility at 0 . This gives an utilitarian welfare of $\frac{k}{2}$ units of utility. However, the optimal utilitarian welfare of $\frac{3 k}{2}$ units of utility locates the facility at 1 . 
We can eliminate the dependency on the tie-breaking rule by having the $k$ agents not at $\frac{1}{2}$ but at $\frac{1}{2}-\epsilon$ for $\epsilon$ tending to zero. $\diamond$

As with minimizing the total distance, the MEdian* mechanism is optimal. No deterministic and strategy proof mechanism has a better approximation guarantee.

Theorem 4 For a facility location problem with limited locations, any deterministic and strategy proof mechanism at best 3-approximates the optimal utilitarian welfare.

Proof: Suppose the only feasible locations for the facility are at 0 or 1 , and there are two agents, one at $\frac{1}{2}-\epsilon$ and the other at $\frac{1}{2}+\epsilon$ for $\frac{1}{2}>\epsilon>0$. There are two cases. In the first case, the mechanism locates the facility at 0 . Suppose the agent at $\frac{1}{2}+\epsilon$ mis-reports their location as 1 . Since the mechanism is strategy proof, the location of the facility cannot change. Consider this new problem with one agent at $\frac{1}{2}-\epsilon$ and the other at 1 . The optimal utilitarian welfare is now $\frac{3}{2}-\epsilon$, but the solution returned by the mechanism has an utilitarian welfare of $\frac{1}{2}+\epsilon$. The approximation ratio is then $\frac{3-2 \epsilon}{1+2 \epsilon}$. As $\epsilon$ approaches zero, this tends to 3 from below. Hence the mechanism at best 3 -approximates the optimal utilitarian welfare. The second case where the facility is located at 1 is symmetric. $\diamond$

\subsection{Egalitarian welfare}

We turn now to the egalitarian welfare. It is not hard to see that the MEDIAN mechanism may not bound the approximation ratio of the optimal egalitarian welfare. Similarly, when the location of facilities is limited, the corresponding MEDIAN* mechanism also may approximate the optimal egalitarian welfare poorly.

Theorem 5 For a facility location problem with limited locations, the MEDIAN* mechanism does not bound the approximation ratio of the optimal egalitarian welfare.

Proof: Suppose facilities can be at $0, \frac{1}{2}$ or 1 . If there are two agents at 0 and one at 1 then the MEDian* mechanism locates the facility at 0 . The egalitarian welfare of this solution has zero units of utility. However, the optimal egalitarian welfare has $\frac{1}{2}$ unit of utility and is achieved by locating the facility at $\frac{1}{2}$. The approximation ratio is therefore unbounded. $\diamond$

In fact, no deterministic and strategy proof mechanism for a facility location problem with limited locations has a bounded approximation ratio. This contrasts with the setting where the facility can be located anywhere and it is possible to show that no deterministic and strategy proof mechanism can do better than $\frac{3}{2}$-approximate the optimal egalitarian welfare, and that the MIDORNEAREST mechanism actually achieves this ratio.

Theorem 6 For a facility location problem with limited locations, no deterministic and strategy proof mechanism has a bounded approximation ratio of the optimal egalitarian welfare.

Proof: Suppose the only feasible locations for the facility are at 0 or 1 , and there are two agents, one at $\frac{1}{4}$ and the other at $\frac{3}{4}$. There are two cases. In the first case, the mechanism 
locates the facility at 0 . Suppose the agent at $\frac{3}{4}$ mis-reports their location as 1 . Since the mechanism is strategy proof, the location of the facility cannot change. Consider this new problem with one agent at $\frac{1}{4}$ and the other at 1 . The optimal egalitarian welfare is now $\frac{1}{4}$ units of utility, but the solution returned by the mechanism has an egalitarian welfare of zero units of utility. The approximation ratio is then unbounded. The second case where the facility is located at 1 is symmetric. $\diamond$

\section{Single facility, satisfaction approximations}

We consider next how well strategy proof mechanisms approximate the optimal social or minimum satisfaction.

\subsection{Social satisfaction}

Mei et al. prove that the MEDIAN mechanism $\frac{3}{2}$-approximates the optimal social satisfaction (Theorem 1 of [10]). In addition, they show that no deterministic and strategy proof mechanism has an approximation ratio of the optimal social satisfaction of less than $8-4 \sqrt{3}$ which is approximately 1.07 (Theorem 3 of [10]). We cannot apply these results directly to our setting as the mechanisms considered in [10] may select an infeasible location for the facility. Instead, we again consider the MEDIAN* mechanism which locates the facility at the nearest feasible location to the median agent.

Theorem 7 For a facility location problem with limited locations, the MEDIAN* mechanism is strategy proof and has an unbounded approximation ratio of the optimal social satisfaction.

Proof: Suppose the only feasible locations for the facility are at 0 or 1 , two agents are at $\frac{1}{2}$, a third agent is at 1 , and the tie-breaking rule locates the facility at 0 . This gives a social satisfaction of zero units of happiness. However, the optimal social satisfaction of 1 unit of happiness requires the facility to be located at 1 . We therefore have an unbounded approximation ratio. Note that we can eliminate the dependency on the tiebreaking rule by having the two agents not at $\frac{1}{2}$ but at $\frac{1}{2}-\epsilon$ for some small $\epsilon>0$ tending to zero. $\diamond$

Unfortunately, we cannot do better. No deterministic and strategy proof mechanism has a bounded approximation ratio of the optimal social satisfaction.

Theorem 8 For a facility location problem with limited locations, no deterministic and strategy proof mechanism has a bounded approximation ratio of the optimal social satisfaction.

Proof: Suppose the only feasible locations for the facility are at 0 or 1 , and there are two agents, one at $\frac{1}{2}-\epsilon$ and the other at $\frac{1}{2}+\epsilon$ for small $\epsilon>0$ that tends to zero. There are two cases. In the first case, the mechanism locates the facility at 0 . Suppose the agent at $\frac{1}{2}+\epsilon$ mis-reports their location as 1 . Since the mechanism is strategy proof, the location of the facility cannot change. Consider this new problem with one agent at $\frac{1}{2}-\epsilon$ and the other at 1 . The solution returned by the mechanism has a social 
satisfaction that tends to zero units of happiness as $\epsilon$ tends to zero. But the optimal social satisfaction is 1 unit of happiness when the facility is at 1 . Hence the mechanism has an unbounded approximation ratio for the optimal social satisfaction. The second case where the facility is located at 1 is symmetric. $\diamond$

\subsection{Minimum satisfaction}

We turn now to the minimum satisfaction. Mei et al. argue that no deterministic and strategy proof mechanism has an approximation ratio of the optimal minimum satisfaction of less than $\frac{4}{3}$ (also Section 3.3 of [10]). The MEDIAN mechanism has an unbounded approximation ratio for the minimum satisfaction. Consider two agents at 0 and one at 1 . Not surprisingly, the MEDIAN* mechanism may also approximate the optimal minimum satisfaction poorly.

Theorem 9 For a facility location problem with limited locations, the MEDIAN* mechanism does not bound the approximation ratio of the optimal minimum satisfaction.

Proof: Suppose facilities can be at $0, \frac{1}{2}$ or 1 . If there are two agents at 0 and one at 1 then the MEDIAN* mechanism locates the facility at 0 . The minimum satisfaction of this solution has zero units of happiness. However, the optimal minimum satisfaction has $\frac{1}{2}$ unit of happiness and is achieved by locating the facility at $\frac{1}{2}$. The approximation ratio is therefore unbounded. $\diamond$

In fact, no deterministic and strategy proof mechanism for a facility location problem with limited locations has a bounded approximation ratio of the minimum satisfaction.

Theorem 10 For a facility location problem with limited locations, no deterministic and strategy proof mechanism has a bounded approximation ratio of the optimal minimum satisfaction.

Proof: Suppose the approximation ratio is bounded by some factor $k$, and the feasible locations for the facility are at $\frac{1}{2}-\frac{1}{k}, \frac{1}{2}-\frac{1}{k}+\frac{1}{k^{2}}, \frac{1}{2}+\frac{1}{k}-\frac{1}{k^{2}}$ or $\frac{1}{2}+\frac{1}{k}$, and there are four agents, one at $\frac{1}{2}-\frac{2}{k}, \frac{1}{2}-\frac{1}{k}+\frac{1}{k^{2}}, \frac{1}{2}+\frac{1}{k}-\frac{1}{k^{2}}$ and the fourth at $\frac{1}{2}+\frac{2}{k}$. Note that if the facility is located at $\frac{1}{2}-\frac{1}{k}$ or $\frac{1}{2}+\frac{1}{k}$ then the minimum satisfaction is zero due to the agents at $\frac{1}{2}+\frac{2}{k}$ and $\frac{1}{2}-\frac{2}{k}$ respectively. Hence, to have a bounded approximation ratio the facility must be located at one of the optimal locations: $\frac{1}{2}-\frac{1}{k}+\frac{1}{k^{2}}$ or $\frac{1}{2}+\frac{1}{k}-\frac{1}{k^{2}}$. There are two cases. In the first case, the facility is located at $\frac{1}{2}-\frac{1}{k}+\frac{1}{k^{2}}$. Suppose the agent at $\frac{1}{2}+\frac{1}{k}-\frac{1}{k^{2}}$ changes their report of their location to 1 . Since the mechanism is strategy proof, the location of the facility must stay the same or more further away from the agent that moves. However, it cannot move further away as the minimum satisfaction would drop to zero and the approximation ratio would be unbounded. Consider this new problem with agents at $\frac{1}{2}-\frac{2}{k}, \frac{1}{2}-\frac{1}{k}+\frac{1}{k^{2}}, \frac{1}{2}+\frac{2}{k}$ and 1 . As $k$ increases, the solution has a minimum satisfaction tending to $\frac{2}{k^{2}}$ units of happiness. But the optimal minimum satisfaction tends to $\frac{4}{k}$ unit of happiness when the facility is at $\frac{1}{2}+\frac{1}{k}-\frac{1}{k^{2}}$. This gives an approximation ratio of $2 k$, contradicting the assumption that the approximation ratio is bounded by some factor $k$. In the second case, the facility is located at $\frac{1}{2}+\frac{1}{k}-\frac{1}{k^{2}}$. The argument is symmetric to the first case. $\diamond$ 


\section{Two facilities, distance approximations}

We move now from locating a single facility to locating two facilities. When facilities are not limited in their location, the only deterministic and strategy proof mechanism for locating two facilities on the line with a bounded approximation ratio for either the optimal total or maximum distance is the ENDPOINT mechanism [13]. This provides a $(n-2)$-approximation of the total distance and a 2-approximation of the maximum distance.

We suppose now that facilities are limited in their location, and consider the corresponding ENDPOINT* mechanism that locates the leftmost facility at the nearest feasible location to the leftmost agent, tie-breaking to the right, and the rightmost facility at the nearest feasible location to the rightmost agent, tie-breaking instead to the left. Tang et al. prove that, when the two facilities are limited in their locations, this mechanism $2 n-3$-approximates the total distance [8]. Tang et al. also prove that the mechanism 3 -approximates the maximum distance, and that no deterministic and strategy proof mechanism can do better [8].

\section{Two facilities, welfare approximations}

When facilities are not limited in their location, the ENDPOINT mechanism offers a good approximation of both the optimal utilitarian and egalitarian welfare. In particular, it is not hard to show that it 2-approximates the optimal utilitarian welfare, and $\frac{3}{2}$ approximates the optimal egalitarian welfare,

When facilities are limited in their locations, the corresponding ENDPOINT* mechanisms provides the same approximation ratio for the utilitarian and egalitarian welfare. We contrast this with the distance objective, where limiting the location of the two facilities worsens the approximation ratio of the ENDPOINT* mechanism. We also contrast this with a single facility, where limiting the location of the facility worsens the approximation ratio for either welfare objective.

Theorem 11 The ENDPOINT* mechanism 2-approximates the optimal utilitarian welfare, and $\frac{3}{2}$-approximates the optimal egalitarian welfare.

Proof: Suppose an agent has utility less than $\frac{1}{2}$. This is only possible iff the agent is served by a facility at the nearest feasible location. Otherwise, an agent has an utility of $\frac{1}{2}$ or greater, compared to a maximum of 1 . Hence, the total of their utilities, the utilitarian welfare is at least half the optimal. Consider $n-2$ agents at $\frac{1}{2}$, and one each at 0 and 1 . Suppose feasible locations are $0, \frac{1}{2}$ and 1 . Then the approximation ratio of the utilitarian welfare is $\frac{2 n-1}{n+2}$. As $n$ goes to infinity, we can make this as close to 2 as we like.

For the egalitarian welfare, there are two cases. In the first case, suppose the agent traveling the furthest to be served is served by the leftmost facility. There is a symmetric argument in the other case for the rightmost facility. There are two subcases. In the first subcase, the leftmost facility is at or to the right of the leftmost agent. The worst case for the approximation ratio has agents at $0, \frac{1}{2}$ and 1 , with $0, \frac{1}{4}$ and 1 being feasible locations for the facilities. The ENDPOINT* mechanism gives an approximation ratio of $\frac{3}{2}$ of the 
optimal egalitarian welfare. In the second subcase, the leftmost facility is to the left of the leftmost agent (as the leftmost agent is at an infeasible location for the facility, and the nearest feasible location is to the left). The worst case for the approximation ratio has the agents at $\frac{1}{4}-\epsilon, \frac{1}{2}$ and 1 , with feasible locations for facilities at $0, \frac{1}{2}$ and 1 , and $\epsilon$ approaching zero. The egalitarian welfare is maximized by locating facilities at $\frac{1}{2}$ and 1. The ENDPOINT* mechanism gives therefore an approximation ratio of $\frac{3}{2} \cdot \diamond$

\section{Two facilities, satisfaction approximations}

When facilities are not limited in their location, the ENDPOINT mechanism offers a good approximation of the social satisfaction but not of the minimum satisfaction. In particular, it is not hard to show that it $\frac{n}{2}-\frac{1}{4}$-approximates the optimal social satisfaction but has no bound on the approximation ratio of the minimum satisfaction.

When facilities are limited in their locations, the corresponding ENDPOINT* mechanism provides a larger approximation ratio for the social satisfaction. We compare this with the distance objective, where limiting the location of the two facilities also worsens the approximation ratio of the ENDPOINT* mechanism. We also compare this with a single facility, where limiting the location of the facility means that the approximation ratio for the social satisfaction becomes unbounded.

Theorem 12 The ENDPOINT* mechanism $\frac{3 n}{4}-\frac{1}{2}$-approximates the optimal social satisfaction, but does not bound the approximation ratio of the optimal minimum satisfaction.

Proof: (Sketch) The worst case for the social satisfaction has facilities at $0, \frac{1}{2}$ or 1 , $n-2$ agents at $\frac{1}{2}$ and at $\frac{1}{4}-\epsilon$ and $\frac{3}{4}+\epsilon$ for $\epsilon$ approaching zero. The optimal facility location with respect to social satisfaction has a facility at $\frac{1}{2}$ and 0 or 1 , giving a social satisfaction tending to $n-\frac{2}{3}$. The ENDPOINT* mechanism locates facilities at 0 and 1 , giving a social satisfaction tending to $\frac{4}{3}$. The approximation ratio is therefore $\frac{3 n}{4}-\frac{1}{2}$.

For the minimum satisfaction, consider agents at $0, \frac{1}{2}$ and 1 with facilities limited to these locations. The ENDPOINT* mechanism locates facilities at 0 and 1, giving a minimum satisfaction of 0 . This compares with a solution with optimal minimum satisfaction that locates a facility at $\frac{1}{2}$, and another at 0 or 1 . The minimum satisfaction is now $\frac{1}{2}$ unit of happiness. Hence the approximation ratio is unbounded. $\diamond$

\section{Conclusions}

We have studied the impact of constraints on the location of a facility on the performance of strategy proof mechanisms for facility location. We considered six different objectives: the total and maximum distance agents must travel, the utilitarian and egalitarian welfare, and the social and minimum satisfaction. In general, constraining facilities to a limited set of locations makes all six objectives harder to approximate in general. For example, a modified median mechanism is strategy proof and 3-approximates both the optimal total and maximum distance. No deterministic and strategy proof mechanism can do better. This contrasts with the setting in which there are no restrictions on where facilities can be located and the median mechanism returns the optimal total distance, and 2-approximates the optimal maximum distance. 


\section{References}

1. Procaccia, A., Tennenholtz, M.: Approximate mechanism design without money. ACM Trans. Econ. Comput. 1(4) (December 2013) 18:1-18:26

2. Golowich, N., Narasimhan, H., Parkes, D.: Deep learning for multi-facility location mechanism design. In: Proceedings of the Twenty-Seventh International Joint Conference on Artificial Intelligence, IJCAI-18, International Joint Conferences on Artificial Intelligence Organization (7 2018) 261-267

3. Drezner, Z., Hamacher, H., eds.: Facility Location: Applications and Theory. Springer (2002)

4. Jagtenberg, C., Mason, A.: Improving fairness in ambulance planning by time sharing. European Journal of Operational Research 280(3) (2020) 1095 - 1107

5. Lu, P., Sun, X., Wang, Y., Zhu, Z.: Asymptotically optimal strategy-proof mechanisms for two-facility games. In: Proceedings of the 11th ACM Conference on Electronic Commerce. EC '10, New York, NY, USA, ACM (2010) 315-324

6. Escoffier, B., Gourvès, L., Thang, N.K., Pascual, F., Spanjaard, O.: Strategy-proof mechanisms for facility location games with many facilities. In Brafman, R., Roberts, F., Tsoukiàs, A., eds.: Algorithmic Decision Theory, Berlin, Heidelberg, Springer Berlin Heidelberg (2011) 67-81

7. Feldman, M., Fiat, A., Golomb, I.: On voting and facility location. In Conitzer, V., Bergemann, D., Chen, Y., eds.: Proceedings of the 2016 ACM Conference on Economics and Computation, (EC '16), ACM (2016) 269-286

8. Tang, Z., Wang, C., Zhang, M., Zhao, Y.: Mechanism design for facility location games with candidate locations. Technical report, CoRR archive within arXiv.org, Cornell University Library (2020)

9. Mei, L., Li, M., Ye, D., Zhang, G.: Strategy-proof mechanism design for facility location games: Revisited (extended abstract). In Jonker, C.M., Marsella, S., Thangarajah, J., Tuyls, K., eds.: Proceedings of the 2016 International Conference on Autonomous Agents \& Multiagent Systems, Singapore, May 9-13, 2016, ACM (2016) 1463-1464

10. Mei, L., Li, M., Ye, D., Zhang, G.: Facility location games with distinct desires. Discrete Applied Mathematics 264 (2019) 148-160

11. Moulin, H.: On strategy-proofness and single peakedness. Public Choice 35(4) (1980) $437-$ 455

12. Massó, J., de Barreda, I.M.: On strategy-proofness and symmetric single-peakedness. Games Econ. Behav. 72(2) (2011) 467-484

13. Fotakis, D., Tzamos, C.: On the power of deterministic mechanisms for facility location games. In Fomin, F., Freivalds, R., Kwiatkowska, M., Peleg, D., eds.: Automata, Languages, and Programming, Berlin, Heidelberg, Springer Berlin Heidelberg (2013) 449-460 